\title{
Latent myeloproliferative neoplasm as a cause of idiopathic splanchnic vein thrombosis: the role of JAK2V6I7F mutation and bone marrow biopsy evaluation
}

\begin{abstract}
Portal vein thrombosis is a common sequela of myeloproliferative neoplasms (MPNs). JAK2V617F is a gain of function mutation that occurs in most patients with polycythemia Vera (PV) and in up to $50 \%$ of patients with essential thrombocythemia (ET) or primary myelofibrosis (PMF). In patients with normal peripheral blood counts, diagnosis of MPN is challenging and requires bone marrow evaluation for formal diagnosis. Testing for JAK2V617F (JAK2) is a useful initial tool to aid in the diagnosis of MPN. In this case report a patient with idiopathic splenic and portal vein thrombosis as well as normal peripheral blood counts is described, who carries a JAK2 mutation and is found to have latent MPN on bone marrow biopsy.
\end{abstract}

Keywords: JAK2V617F (JAK2) mutation, myeloproliferative neoplasms, splanchnic veins thrombosis
Volume I Issue 4 - 2015

\author{
Oksana Volod \\ Department of Pathology and Laboratory Medicine, Cedars \\ Sinai Medical Center, USA
}

\begin{abstract}
Correspondence: Oksana Volod, Department of Pathology and Laboratory Medicine, Cedars Sinai Medical Center, Los Angeles, CA, USA, Tel (3I0) 423-547I, Fax (310) 423-0483, Email Oksana.Volod@cshs.org
\end{abstract}

Received: October 31,2015 | Published: November 18, 2015
Abbreviations: MPN, myeloproliferative neoplasms; PV, polycythemia vera; ET, essential thrombocythemia; PMF, primary myelofibrosis; CBC, complete blood count; SVT, splanchnic veins thrombosis; PVT, portal vein thrombosis; BCS, budd chiari syndrome; AST, aspartate amino transferase; ALT, alanine aminotransferase; WBC, white blood cell count; CT, computed tomography; Ig, immunoglobulin; ADP, adenosine diphosphate; PFA-100, platelet function analyzer; Epi, epinephrine; INR, international normalized ratio; $\mathrm{CD}$, cluster of differentiation; $\mathrm{BCR}-\mathrm{ABL}$, breakpoint cluster region-abelson; AML, acute myelogenous leukemia; vWD, von willebrand disease; $\mathrm{LDH}$, lactate dehydrogenase

\section{Introduction}

Splanchnic veins thrombosis (SVT) includes the portal vein thrombosis (PVT) and the primary Budd-Chiari syndrome (BCS). These are sites of so called "unusual thrombosis". Budd-Chiari syndrome is caused by complete or partial obstruction of hepatic venous outflow tract. BCR- ABL negative myeloproliferative neoplasms (MPNs) are the most frequent hypercoagulable risk factor in both BCS (prevalence 30\%-50\%) and PVT (prevalence $15 \%-30 \%){ }^{1}$ However, in at least $25 \%$ of cases, when no obvious cause can be identified and complete blood count (CBC) is normal, a latent myeloproliferative neoplasm (MPN) should be considered. Although the mechanisms involved have not been fully defined, portal hypertension and its complications (splenomegaly, hemodilution and iron deficiency) can account for complete blood count to be within normal ranges. Latent MPN has been detected in $25-65 \%$ of patients with SVT. ${ }^{2}$ This case report describes splenic vein and portal vein thrombosis (SPVT) in a patient as the first presenting symptom of latent MPN. On further workup JAK2 mutation was detected and bone marrow biopsy was consistent with the diagnosis of latent MPN. Extensive hypercoagulable work up was essentially negative in this patient. A role of the testing for JAK2V617F and a bone marrow biopsy evaluation in patients with idiopathic SVT will be discussed.
Other acquired coagulopathies seen in MPN and their laboratory diagnostic work up will be reviewed.

\section{Case presentation}

A 47 year old African-American male with no history of known medical problems presented with four days of left upper quadrant abdominal pain associated with bloating and constipation. He denied any nausea, vomiting, weight loss, night sweats and any history of alcohol or other drug abuse. In the emergency room his labs revealed mild transaminitis (AST 96U/L, ALT 58U/L), hyperbilirubinemia (total bilirubin 2.1MG/DL) and pancreatitis (amylase 116U/L, lipase 100U/L). Complete blood CBC showed normal white blood cell count (WBC) $(10.3 \times 1000 / \mathrm{UL})$, hemoglobin $(15.6 \mathrm{~g} / \mathrm{dl})$, hematocrit $(47.8 \%)$, and platelet count $\left(292 \times 10^{9} / \mathrm{L}\right)$. Abdominal ultrasound showed no common bile duct dilatation, gallstones, or cirrhosis. CT scan of the abdomen was significant for occlusions in the right hepatic branches of the intra hepatic portal vein, main portal vein, and splenic vein. No masses were noted in the liver.

Gastroenterology was consulted given new findings of SPVT. Esophagogastroduodenoscopy showed non-bleeding gastric varices likely secondary to splenic vein thrombosis. No evidence of colorectal cancer was visualized on colonoscopy. Endoscopic ultrasound revealed normal pancreatic parenchyma with no masses or lesions. Magnetic resonance imaging cholangiogram (MRCP) showed massive splenomegaly measuring $19 \mathrm{~cm}$ but no intra or extrahepatic biliary ductal dilatation or pancreatic ductal dilatation.

Coagulation service was consulted next for evaluation of a possible pro-thrombotic state to explain his SPVT, as there was insufficient evidence to attribute the cause of his thrombosis to chronic pancreatitis. Tests used to screen for a pro-thrombotic state including prothrombin G20210A gene mutation, anti-phospholipid antibodies (lupus anticoagulant, anti-cardiolipin IgG and IgM, anti-b2 GPI IgG and IgM and phosphatidylserine IgG and IgM antibodies), genotyping 
for Factor V Leiden, anti-thrombin 3level, protein C functional assay, plasminogen level and flow cytometry for paroxysmal nocturnal hemoglobinuria $(\mathrm{PNH})$ were all unremarkable. Factor VIII level $(516 \%)$ and fibrinogen $(622 \mathrm{mg} / \mathrm{dl})$ which are acute phase reactants were elevated. Protein S activity assay was decreased at $35 \%$ as expected given the patient's known recent SPVT, presence of acute phase reactants and warfarin therapy initiation.

Peripheral blood smear was significant for megathrombocytes (Figure 1). Platelet aggregometry studies with high and low doses of epinephrine and ADP and spontaneous aggregation (hyperaggregable profile), ristocetin induced platelet aggregation (RIPA), arachidonic acid and collagens were normal showing no evidence of platelet hyperaggregability, platelet qualitative defect or suggestion of MPN. Primary hemostasis screening assay PFA-100 was normal with both PFA Epi $121 \mathrm{sec}(65-185 \mathrm{sec})$ and PFAADP $104 \mathrm{sec}(71-118 \mathrm{sec})$ closure times. Given these findings as well as clinic presentation with SPVT, the patient was screened for JAK2 mutation with results pending at time of discharge. The patient was started on a heparin infusion while hospitalized and eventually discharged on warfarin.

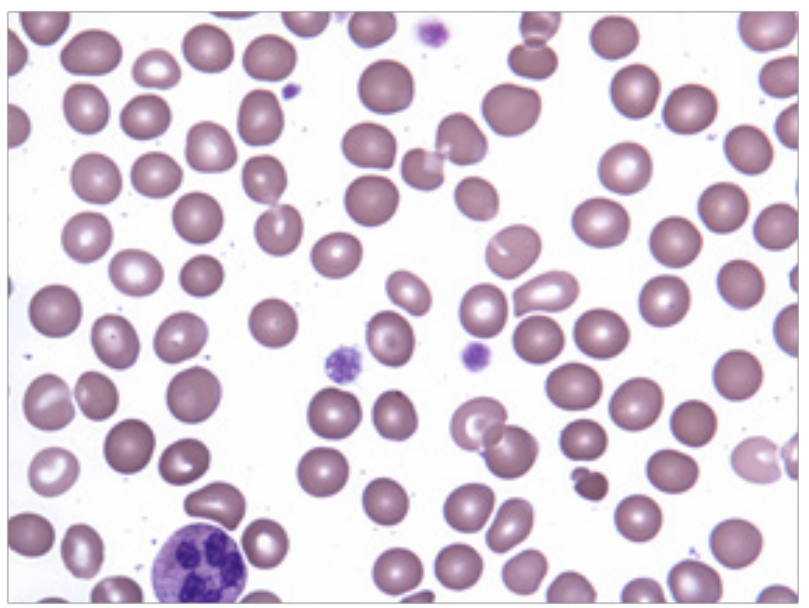

Figure I Peripheral blood smear with large platelets (megathrombocytes).

Approximately one month after discharge, the patient was readmitted with recurrent abdominal pain. Repeat CT scan showed stable thrombosis from prior admission as well as a new splenic infarct. Of note, the patient's INR was noted to be subtherapeutic at 1.2 despite adherence to warfarin. He was subsequently restarted on a heparin infusion. During this admission, the patient was also noted to be positive for clonal JAK2 V617F activation mutation.

The patient eventually underwent bone marrow biopsy which showed a hypercellular marrow with atypical megakaryocytic hyperplasia (Figure 2) and mild reticulin fibrosis (Figure 3). By immunohistochemistry and flow cytometric analysis CD34-positive blasts were not increased. Overall morphologic findings, in association with the positive JAK2 mutation were most consistent with early MPN. Molecular studies for BCR-ABL gene rearrangement were negative. The patient was advised to remain on lifelong anticoagulation. Subsequently, within the year, his red blood cell count started to rose to around $6 \mathrm{MILL} / \mathrm{UL}$ (3.97-5.65MILL/UL), hemoglobin to 18G/ DL (13-17G/DL), hematocrit to $53 \%(37.5-49.9 \%)$ but his platelet and leukocyte count remained normal and he was diagnosed with polycythemia Vera (PV). Currently the patient is managed with intermittent phlebotomy and anticoagulation with warfarin and for the last 5 years he had no symptoms of MPN progression.



Figure 2 Hypercellular marrow with atypical megakaryocytic hyperplasia

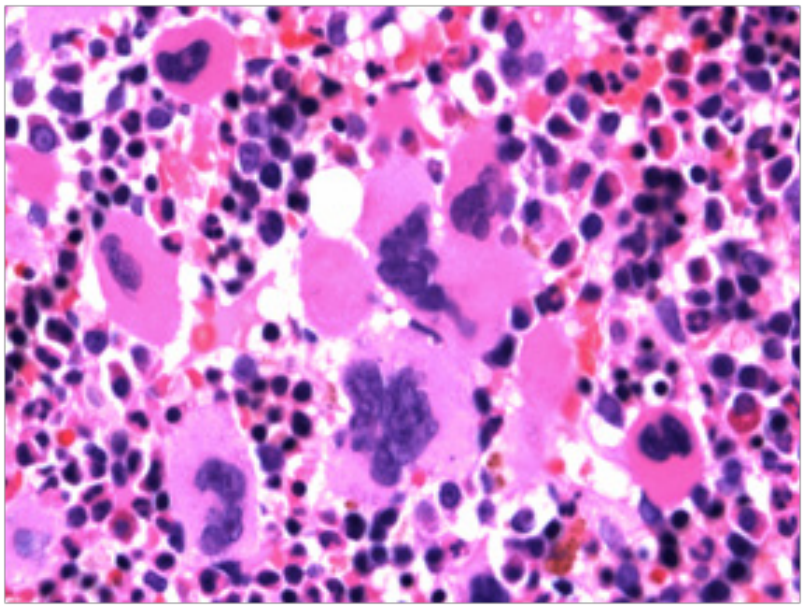

Figure 3 Megakaryocytes clustering in marrow.

\section{Discussion}

The myeloproliferative neoplasms (MPNs), previously termed the myeloproliferative disorders, are characterized by the clonal proliferation of one or more hematopoietic cell lineages, predominantly in the bone marrow, but sometimes in the liver and spleen. The classic MPNs include polycythemia Vera (PV), essential thrombocythemia (ET), and primary myelofibrosis (PMF). Absence of the BCR-ABL gene rearrangement, caused by a reciprocal translocation between chromosomes 9 and 22, distinguishes these entities from other myeloproliferative neoplasms such as CML. Classification of MPNs is based on 2008 World Health Organization criteria which incorporates clinical data, peripheral blood count abnormalities, histological features of bone marrow and the use of genetic markers. ${ }^{3}$

\section{Diagnosis}

PV is characterized by increased red blood cell proliferation that has become independent of normally regulated erythropoiesis. Patients may present with headaches, dizziness, visual disturbances, pruritus and erythromelalgia. Thrombosis, hemorrhage and progression to acute myelogenous leukemia may complicate the course of disease. $\mathrm{PV}$ is more prevalent in BCS than in PVT. ${ }^{1}$ More than $95 \%$ of patients with PV carry The JAK2 mutation, making it a critical component in diagnosis. Diagnosis of PV requires the presence of both major criteria and one minor criterion or the presence of the first major criterion together with two minor criteria. 
Major WHO criteria are as follows:

i. Hemoglobin $>18.5 \mathrm{~g} / \mathrm{dL}$ in men and $>16.5 \mathrm{~g} / \mathrm{dL}$ in women, or other evidence of increased red blood cell volume.

ii. Presence of JAK2V617F or other functionally similar mutation, such as JAK2 exon 12 mutation.

iii. Minor WHO criteria are as follows:

iv. Bone marrow biopsy exhibiting hypercellularity with trilineage (erythroid, granulocytic and megakaryocytic) hyperplasia.

v. Low serum erythropoietin level.

vi. Endogenous erythroid colony formation in vitro.

As with PV, ET can be complicated by life threatening thromboembolic and hemorrhagic events. Hemorrhagic episodes are due to acquired von Willebrand disease (vWD). More than $50 \%$ of patients are asymptomatic at the time of diagnosis. Less than $5 \%$ of patients progress to acute myelogenous leukemia (AML). Diagnosis of ET requires the presence of all major criteria.

Major WHO criteria are as follows:

i. Persistent platelet counts $>450 \times 10^{9} / \mathrm{L}$.

ii. Bone marrow core biopsy revealing predominantly large megakaryocytes with hyperlobulated nuclei dispersed throughout the marrow or in loose clusters (Figure 4).

iii. Not meeting WHO criteria for other MPN, myelodysplastic syndrome or other myeloid neoplasm.

iv. Presence of JAK2V617F or other functionally clonal mutation or no evidence of reactive

v. thrombocytosis.

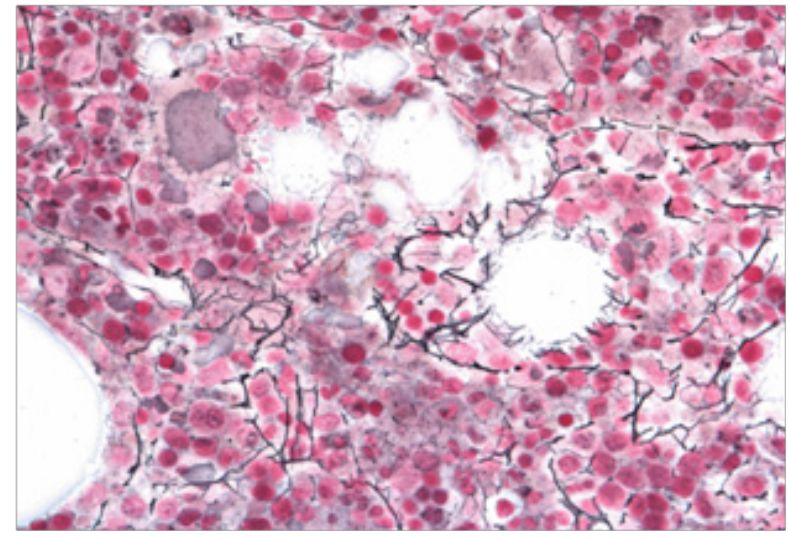

Figure 4 Increased reticulin in marrow (reticulin stain).

The JAK2 mutation is less specific for ET since it is present in only 40 to 50percent of ET cases. However, when present, JAK2 excludes reactive thrombocytosis. PMF is a progressive disease characterized by predominantly megakaryocytes and granulocytes in the early stages of marrow involvement with eventual development of reticulin and/or collagen fibrosis and eventual osteosclerosis. Thirty percent of patients are asymptomatic at time of diagnosis; disease is suspected when a patient presents with splenomegaly or peripheral blood counts revealing anemia, leukocytosis and or thrombocytosis. Bone marrow failure, thromboembolic disease, AML is major causes of morbidity and mortality. Bone marrow biopsy provides not only diagnostic information but also clues for prognostication. Higher grades of bone marrow fibrosis indicate more advanced disease and poor prognosis. Diagnosis of PMF requires the presence of all 3 major criteria and 2 minor criteria.

Major WHO criteria are as follows:

i. Bone marrow biopsy exhibiting megakaryocytes proliferation and atypia accompanied by fibrosis

ii. Not meeting WHO criteria for other MPN, myelodysplastic syndrome or other myeloid neoplasm

iii. Presence of JAK2V617F or other clonal marker or no evidence of reactive marrow fibrosis

Minor WHO criteria are as follows:

i. Leukoerythroblastosis

ii. Increased serum lactate dehydrogenase (LDH)

iii. Anemia

iv. Palpable splenomegaly

\section{Risk of bleeding and clotting stratification}

Once diagnosis is established, patient should be evaluated for other potential thrombotic or hemorrhagic manifestations of MPN which are the main causes of mortality and morbidity in patients with MPN. Vascular thrombotic events are both arterial and venous, and may occur virtually in any area. Most thrombotic events occur at or in the two years before diagnosis. ${ }^{4}$

Multiple factors are likely to contribute to the pathogenesis of thrombosis. Platelet hyper reactivity is one of them. ${ }^{5}$ Platelet aggregometry may be useful tool to demonstrate enhanced platelet activation and may help select MPN patients for aspirin therapy. Our patient has normal platelet function with no evidence of hyperactive platelets. If patient is tested for prothrombotic state during an acute event or when on anticoagulation therapy, it is important that clinicians appreciate that tests results for protein $\mathrm{C}$, protein $\mathrm{S}$, anti-thrombin III, factorVIII, fibrinogen and lupus anticoagulant can be affected, so that they are not misinterpreted.

The hemorrhagic manifestation is usually observed in patients with thrombocytosis due to acquired von Willebrand disease (vWD) and not due to impaired platelet function. ${ }^{6}$ Aspirin given to such a patient may results in severe bleeding complications and is not advocated for patients with bleeding, even if platelet count is high. Therefore patient with bleeding history should be screened for vWD with primary hemostasis PFA-100 assays. PFA-100 has a reported sensitivity of $95 \%$ in detecting abnormalities of primary hemostasis such as platelet dysfunction, drug effect, uremia and vWD. If patient history/physical examination gives a strong indication of a bleeding disorder, von Willebrand disease panel is recommended to complete work up. In our patient with no history of bleeding and normal PFA100 , vWD work up was not warranted.

\section{Management of MPN}

Current treatments indications for PV and ET are risk oriented and are derived from randomized clinical trials performed within national or international collaborative groups. ${ }^{7-10}$ 
PV management: Patients with low (age $<60 y$ and no prior cardiovascular event) and intermediate (generic cardiovascular risk factors) risk categories are managed with phlebotomies and low dose aspirin. Patients with high (age $>60 \mathrm{y}$ and or prior cardiovascular event) risk category are managed with myelosuppression $+/$ - phlebotomies and low dose aspirin.

ET management: Low - risk patients with asymptomatic ET either do not need therapy or low dose aspirin is recommended (no consensus).

i. Patients with intermediate risk category can be managed with low dose aspirin (no consensus).

ii. Patients with high risk category are managed with myelosuppression and low dose aspirin.

Extreme thrombocytosis is considered a contraindication for aspirin therapy due to acquired von Willebrand disease. ${ }^{6,11}$

PMF management: Allogeneic stem cell transplant is the only approach that has resulted in prolongation of survival in patients with PMF. It is usually reserved for patients with high risk disease. ${ }^{12}$

\section{Role of molecularly targeted therapy in MPN}

Ruxolitinib, a selective JAK-1 and 2inhibitor, is effective in controlling splenomegaly and constitutional symptoms, but has a limited benefit in reversing bone marrow fibrosis or inducing remissions in PMF. It is currently in Phase 3 testing for treatment of hydroxyurea resistant/intolerant $\mathrm{PV} .{ }^{13}$

\section{Conclusion}

In the absence of obvious abnormal peripheral blood counts, diagnosis of MPN is often challenging. Latent MPN has been detected in $25-65 \%$ of patients with SVT and should be considered in differential diagnosis of patients presenting with idiopathic SVT. The presented case shows that screening for JAK2V617F mutation is an important tool for detecting MPNs in a patient with newly diagnosed idiopathic SVT and should be included in the thrombophilia work up. If mutation is detected, patient should be thoroughly investigated for evidence of MPNs. Characterization of acquired coagulopathy (hyperaggregable platelets vs acquired vWD) is necessary for the proper anticoagulation management. The subgroup of JAK2 positive patients without evidence of MPN require close monitoring and long term follow up for future development of MPNs.

\section{Acknowledgements}

None.

\section{Conflict of interest}

The author declares no conflict of interest.

\section{References}

1. Smalberg JH, Arends LR, Valla DC, et al. Myeloproliferative neoplasms in Budd-Chiari syndrome and portal vein thrombosis: a meta-analysis. Blood. 2012;120(25):4921-4928.

2. Regina S, Herault O, D'Alteroche L, et al. JAK2 V617F is specifically associated with idiopathic splanchnic vein thrombosis. J ThrombHaemost. 2007;5(4):859-861.

3. Swerdlow SH, Campo E, Harris NL, et al. WHO classification of Tumours of Haematopoietic and Lymphoid tissues. World Health Organisation. 2008;2(2)

4. Policitemia GIS. Polycythemia vera: the natural history of 1213 patients followed for 20 years. Gruppo Italiano studio policitemia. Ann Intern Med. 1995;123:656-664.

5. Manoharan A, Gemmell R, Brighton T, et al. Thrombosis and bleeding in myeloproliferative disorders: identification of at-risk patients with whole blood platelet aggregation studies. Br J Haematol. 1999;105:618-625.

6. Kumar S, Pruthi RK, Nichols WL. Acquired von Willebrand disease. Mayo Clin Proc. 2002;77(2):181-187.

7. Berk PD, Goldberg JD, Donovan PB, et al. Therapeutic recommendations in polycythemia vera based on Polycythemia Vera Study Group protocols. SeminHematol. 1986;23(2):132-143.

8. Marchioli R, Finazzi G, Landolfi R, et al. Vascular and neoplastic risk in a large cohort of patients with polycythemia vera. J Clin Oncol. 2005;23(10):2224-2232.

9. Fruchtman SM, Mack K, Kaplan ME, et al. From efficacy to safety: a Polycythemia Vera Study group report on hydroxyurea in patients with polycythemia vera. SeminHematol. 1997;34(1):17-23.

10. Harrison CN, Campbell PJ, Buck G, et al. Hydroxyurea compared with anagrelide in high-risk essential thrombocythemia. $N$ Engl J Med. 2005;353(1):33-45.

11. Kessler CM. Propensity for hemorrhage and thrombosis in chronic myeloproliferative disorders. SeminHematol. 2004;41(Suppl 2):10-14.

12. Kroger N, Mesa RA. Choosing between stem cell therapy and drugs in myelofibrosis. Leukemia. 2008;22(3):474-486.

13. Pardanani A, Tefferi A. Is there a role for JAK inhibitors in BCR-ABL1negative myeloproliferative neoplasms other than myelofibrosis? Leuk Lymphoma. 2014;55(12):2706-2711. 